\title{
Control of femtosecond laser interference ejection with angle and polarisation
}

David M. Roper, Stephen Ho, Moez Haque, Peter R. Herman

David M. Roper, Stephen Ho, Moez Haque, Peter R. Herman, "Control of femtosecond laser interference ejection with angle and polarisation," Proc. SPIE 10094, Frontiers in Ultrafast Optics: Biomedical, Scientific, and Industrial Applications XVII, 100940T (15 March 2017); doi: 10.1117/12.2256995 


\title{
Control of Femtosecond Laser Interference Ejection with Angle and Polarization
}

\author{
David M. Roper*, Stephen Ho, Moez Haque, Peter R. Herman \\ Department of Electrical and Computer Engineering, 10 King's College Rd., University of Toronto \\ Toronto,Ontario,M5S3G4,Canadadavid.roper@mail.utoronto.ca; stephen.ho@utoronto.ca; \\ moez.haque@mail.utoronto.ca; p.herman@utoronto.ca
}

\begin{abstract}
The nonlinear interactions of femtosecond lasers are driving multiple new application directions for nanopatterning and structuring of thin transparent dielectric films that serve in range of technological fields. Fresnel reflections generated by film interfaces were recently shown to confine strong nonlinear interactions at the Fabry-Perot fringe maxima to generate thin nanoscale plasma disks of 20 to $40 \mathrm{~nm}$ thickness stacked on half wavelength spacing, $\lambda / 2 n_{\text {film }}$, inside a film (refractive index, $\mathrm{n}_{\text {film}}$ ). The following phase-explosion and ablation dynamics have resulted in a novel means for intrafilm processing that includes 'quantized' half-wavelength machining steps and formation of blisters with embedded nanocavities. This paper presents an extension in the control of interferometric laser processing around our past study of $\mathrm{Si}_{3} \mathrm{~N}_{4}$ and $\mathrm{SiO}_{\mathrm{x}}$ thin films at $515 \mathrm{~nm}, 800 \mathrm{~nm}$, and $1044 \mathrm{~nm}$ laser wavelengths. The role of laser polarization and incident angle is explored on fringe visibility and improving interferometric processing inside the film to dominate over interface and / or surface ablation. $\mathrm{SiO}_{\mathrm{x}}$ thin films of $1 \mu \mathrm{m}$ thickness on silicon substrates were irradiated with a $515 \mathrm{~nm}$ wavelength, 280 fs duration laser pulses at $0^{\circ}$ to $65^{\circ}$ incident angles. A significant transition in ablation region from complete film removal to structured quantized ejection is reported for $\mathrm{p}$ - and s-polarised light that is promising to improve control and expand the versatility of the technique to a wider range of applications and materials. The research is aimed at creating novel bio-engineered surfaces for cell culture, bacterial studies and regenerative medicine, and nanofluidic structures that underpin lab-in-a-film. Similarly, the formation of intrafilm blisters and nanocavities offers new opportunities in structuring existing thin film devices, such as CMOS microelectronics, LED, lab-on-chips, and MEMS.
\end{abstract}

Keywords: Femtosecond laser processing, thin optical film, interferometric processing, nanostructured film, intrafilm processing

\section{INTRODUCTION}

In a rapidly developing research and industrial marketplace, laser processing of dielectric films presents an opportunity for single step fabrication and dramatically reduced production times during nano- and micro- scale device manufacturing. The understanding and control of processing methods for thin transparent films is beneficial to the further advancement of integrated optics, display, lab-on-a-chip, and micro-electromechanical sensors. Femtosecond lasers are particularly well suited to drive non-linear interactions inside transparent materials, where the absorption can be localised to small focal volumes that enables manufacturing of waveguides [1,2], Bragg gratings $[\mathbf{3}, \mathbf{4}]$, couplers [5], filters and a wide range of optical sensors [6] at arbitrary three-dimensional (3D) positions within the bulk material. However, when focusing into a transparent film, the beam confocal parameter typically exceeds the film thickness by multiple factors to enable competing interaction dynamics to be driven from the substrate and interfaces. A diverse range of applications have emerged from this direction, for example, from laser interaction physics centred at the film-substrate interface that fully eject the film to enable the patterning of conductive coatings on display glass [7], or the printing of

Frontiers in Ultrafast Optics: Biomedical, Scientific, and Industrial Applications XVII, edited by Alexander Heisterkamp, Peter R. Herman, Michel Meunier, Roberto Osellame, Proc. of SPIE Vol. 10094, 100940T · C 2017 SPIE - CCC code: 0277-786X/17/\$18 · doi: 10.1117/12.2256995 
novel electronic, biological, and mesoscopic structures by laser-induced forward transfer (LIFT) [8]. Alternatively, weaker laser exposures can selectively de-laminate the film and form micro- or nano-channel devices along a silicasilicon interface $[\mathbf{9 , 1 0}]$.

Increasing control over surface topography and feature formation is desired for additional refinement and tailoring of deposited thin-films to improve performance. Whilst modification of thin films at the top surface and through interface interactions is well established $[\mathbf{9 , 1 0}]$, intra-film processing is only a recent promising development that relies on Fresnel reflections at the film surfaces to drive strong and high confined nonlinear interference effects inside the film. In this way, Kumar et al. [11,12] demonstrated quantized structuring in sub-micron $\mathrm{Si}_{3} \mathrm{~N}_{4}$ thin-films on silicon substrates, generating thin nano-plasma disks that open nanocavities and thin blisters from within the film. This phenomenon was extended to multiple laser wavelengths in $\mathrm{Si}_{3} \mathrm{~N}_{4}$ films [13] and to 1 and $3.5 \mu \mathrm{m}$ thick $\mathrm{SiO}_{\mathrm{x}}$ films by Ho et al. [14]. Here, for the first time, we consider the effects of laser incident angle on light confinement within the $\mathrm{SiO}_{\mathrm{x}}$ film, manipulating the interface reflection with s- and p- polarisation to regulate the contrast and spacing of interference fringes. In this way, we report the benefits for improving control of intra-film quantized ejection.

\section{BACKGROUND}

Confinement of strong nonlinear interactions at Fabry-Perot fringe maxima is principally controlled by the optical reflectance at the film-substrate interface [11]. Fresnel reflection amplitudes for s- and p- polarized light depend on the refractive index contrast between the $\mathrm{SiO}_{\mathrm{x}}$ film $\left(n_{\text {film }}=1.46\right)$ and silicon substrate $\left(n_{\text {substrate }}=4.22+0.038 \mathrm{i}\right)$, and vary strongly with the angle of the laser incident at the interface $\left(\theta_{i}\right)$, and the corresponding refractive angle into the substrate $\left(\theta_{t}\right)$, according to:

$$
\begin{aligned}
& r_{s}=\frac{n_{\text {film }} \cos \left(\theta_{i}\right)-n_{\text {substrate }} \cos \left(\theta_{t}\right)}{n_{\text {film }} \cos \left(\theta_{i}\right)+n_{\text {substrate }} \cos \left(\theta_{t}\right)} \\
& r_{p}=\frac{n_{\text {film }} \cos \left(\theta_{t}\right)-n_{\text {substrate }} \cos \left(\theta_{i}\right)}{n_{\text {film }} \cos \left(\theta_{t}\right)+n_{\text {substate }} \cos \left(\theta_{i}\right)}
\end{aligned}
$$

At normal incidence, the reflection coefficients are polarization independent and yield a moderately strong fringe visibility contrast of 0.78 for $\mathrm{SiO}_{\mathrm{x}}$ thin film on a silicon substrate for $515 \mathrm{~nm}$ wavelength light [14]. Sufficient interference contrast exists to drive nonlinear absorption and formation of thin plasma disks with 20 to $40 \mathrm{~nm}$ thickness [11]. We have previously shown that this contrast is sufficient to open intra-film nanocavities that align with the interference fringe maxima and, with sufficiently high pulse energies, drive quantized ejections on half-wavelength spacing of $\lambda / 2 n_{\text {film }}$. For example, Figure 1 shows scanning electron micrographs (SEM) of single-pulse irradiation spots in $3.5 \mu \mathrm{m} \mathrm{SiO}_{\mathrm{x}}$ film, where terraced ejections of half-wavelength film segments have been opened with a Gaussian beam spot [14]. With a systematic reduction in fluence, the number of ejected segments is seen to decrease in a controlled manner from 6 segment ejection at $99 \mathrm{~J} / \mathrm{cm}^{2}$ (top left) to 3 segment ejection at $3.5 \mathrm{~J} / \mathrm{cm}^{2}$ (bottom right) fluence exposure. 


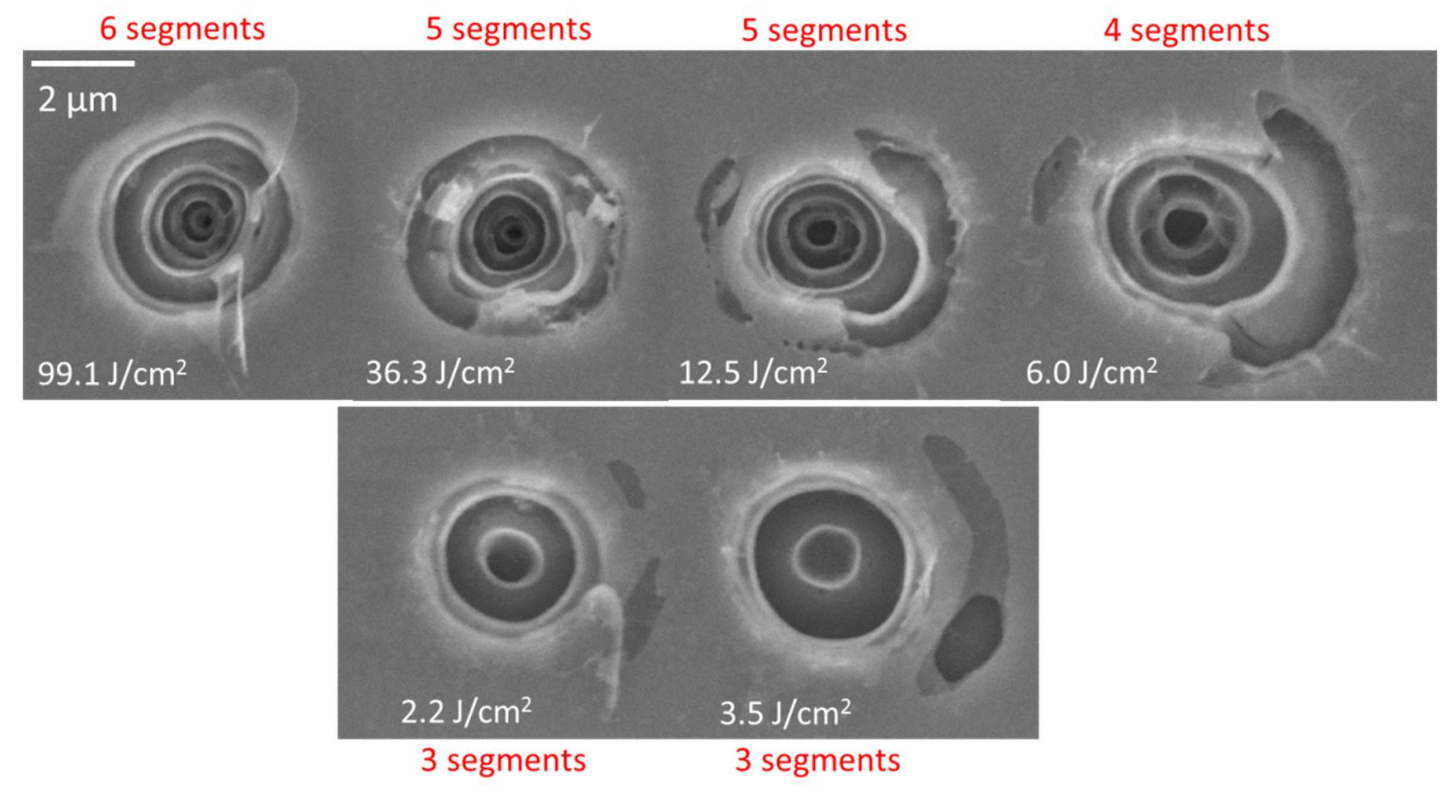

Figure 1: SEM of $3.5 \mu \mathrm{m}$ thick $\mathrm{SiO}_{\mathrm{x}}$ film on silicon, irradiated with single femtosecond laser pulses of $515 \mathrm{~nm}$ wavelength at decreasing fluence exposures of $99.1 \mathrm{~J} / \mathrm{cm}^{2}, 12.5 \mathrm{~J} / \mathrm{cm}^{2}, 6.0 \mathrm{~J} / \mathrm{cm}^{2}$, and $2.2 \mathrm{~J} / \mathrm{cm}^{2}$, demonstrating quantized intra-film ejections of $3,4,5$, and 6 segments respectively.

The schematics in Figure 2 show laser light incident on $1 \mu \mathrm{m}$ thick $\mathrm{SiO}_{\mathrm{x}}$ film and $\mathrm{Si}$ substrate at conventional geometry of normal incidence in (a) and at a sample tilt of $\theta_{1}$ in (b). At normal incidence, a total of 6 intensity peaks are expected in the interference pattern, presenting a moderately strong visibility contrast of 0.78 as shown in the graph. At an increased incident angle and reflection at the interface in Figure 2(b), beam overlap in the film will still generate interference fringes. However, the fringe spacing expands and the visibility contrast becomes polarization sensitive as indicated in the s- and p-polarization intensity plots on the left, calculated for $\theta_{1}=65^{\circ}$. The fringe spacing has increased by $27 \%$ from $180 \mathrm{~nm}$ to $230 \mathrm{~nm}$. For s-polaraization, the visibility contrast has increased by $10 \%$ from $0.78\left(\theta_{1}=0\right)$ to $0.85\left(\theta_{1}=65^{\circ}\right)$. Hence, the film intensity distribution calculated for s-polarized light is very similar to the case of normal incidence in Figure 1(a), suggesting a high degree of laser interaction confinement. Clear ejections of half-wavelength film segments are to be expected, as was observed in Figure 1 for a normal incidence irradiated $\mathrm{SiO}_{\mathrm{x}}$ film. In contrast, The fringe visibility contrast for p-polarized light has decreased more than 5-fold from 0.79 at normal incidence to 0.15 at $\theta_{1}=65^{\circ}$, predicting formation of more diffuse plasma disks in the film, potentially falling below the threshold for quantized intra-film etching. In this paper, the polarization dependence for quantized ejection of $1 \mu \mathrm{m} \mathrm{SiO}_{\mathrm{x}}$ file is studied at a fixed angle of $\theta_{1}=65^{\circ}$, where we seek to manipulate the visibility contrast of fringes over the expected 6 fold increase between $\mathrm{p}$ - and s- polarized light. This dramatic difference is expected to show quantized intra-film processing for s-polarized light and full film ejection for p-polarized light. 


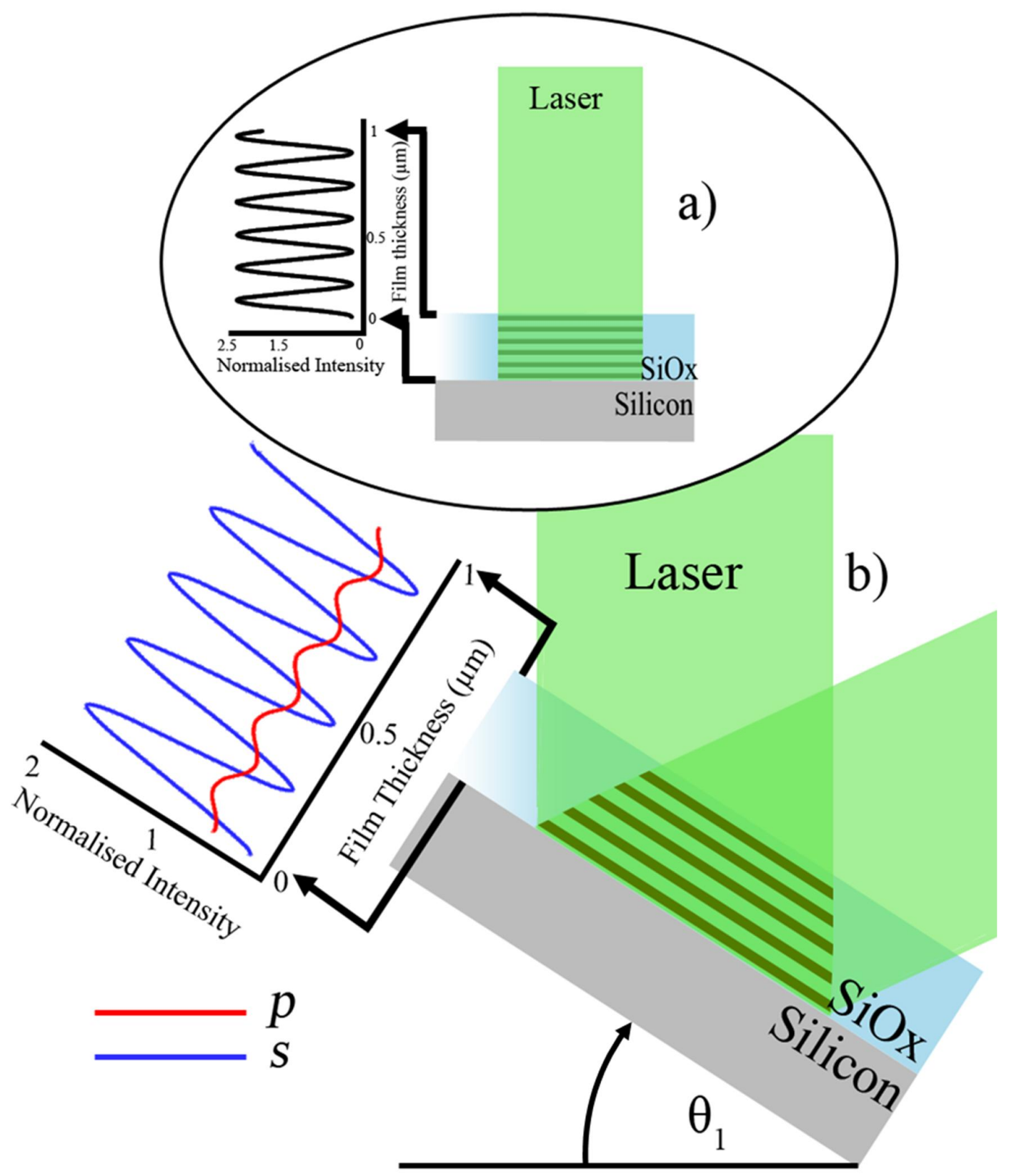

Figure 2: a) shows the laser exposure geometry of normal incidence on a thin $\mathrm{SiO}_{\mathrm{x}}$ film on a silicon substrate, and the associated intensity interference distribution of green light, $515 \mathrm{~nm}$ wavelength, in the $1.0 \mu \mathrm{m}$ thick film. At an incident angle of $\theta_{l}$, leading to an internal film angle $\theta_{i}$ b) overlapping beam reflections in the $1 \mu \mathrm{m} \mathrm{SiO} \mathrm{O}_{\mathrm{x}}$ film form fringes on wider spaced $\lambda / 2 \cos \left(\theta_{i}\right) n_{\text {film }}$, with intensity distribution in the thin film having reduced fringe visibility for p-polarization (red), compared with stronger for s-polarization (blue). 


\section{MATERIALS AND EXPERIMENTAL METHODS}

Thin films of $\mathrm{SiO}_{\mathrm{x}}$ were grown to $1.0 \mu \mathrm{m}$ thickness over substrates of polished phosphorus-doped (001) crystalline silicon wafers of $400 \mu \mathrm{m}$ thickness by plasma-enhanced chemical vapour deposition. Wafers were diced into $1 \mathrm{~cm}^{2}$ samples and cleaned with lens tissue soaked in a dilute ethanol solution. Adhering samples to microscope slides permitted flexible mounting for laser processing and diagnostic (Optical and Scanning Electron Microscopy) tools. Analysis by SEM (Hitachi SU-8320) at a $30^{\circ}$ sample tilt was required for higher resolution imaging. A lower secondary electron detector provided topography dominated images which best identified evidence of quantized ejection.

A fibre-amplified laser system (Amplitude Systemes, Satsuma) with linearly polarized and Gaussian-shaped pulses $\left(\mathrm{M}^{2}=\right.$ 1.14 of $1030 \mathrm{~nm}$ wavelength, frequency doubled to $515 \mathrm{~nm}$ ) wasapplied to films with pulse energies varied from $10 \mathrm{~nJ}$ to $1700 \mathrm{~nJ}$. Translational motion was controlled under a focusing lens via programmable 3-axis motion stages (Aerotech ABL1000) to sub-micron alignment precision, while an angle mount with single axis rotation of $0^{\circ}$ to $90^{\circ}$ provided control of sample orientation, $\theta_{l}$, with respect to the incident laser beam. Polarization state was manipulated with a $90^{\circ}$ rotation of the sample mount azimuthally about the optical path. Further details of the laser arrangement can be found in Haque et al. $[\mathbf{1 5}, \mathbf{1 6}]$.

For normal incidence $\left(\theta_{I}=0^{\circ}\right)$, the sample tilt was aligned to orthogonally reflect an attenuated and focussed laser beam, a whilst focal spot alignment to the film surface was confirmed by imaging the reflected beam via a split mirror onto a CCD camera. A $30 \mu \mathrm{m}$ by $60 / \cos \left(\theta_{1}\right) \mu \mathrm{m}$ spaced grid of single-pulse exposure spots were generated by various pulse energies and focal displacements to provide a range of fluence and spot size exposures on the sample films. Axial focal spot displacements of $5 \mu \mathrm{m}$ increments were tested from $200 \mu \mathrm{m}$ below to $200 \mu \mathrm{m}$ above the best focus position.

\section{Focal Displacement}

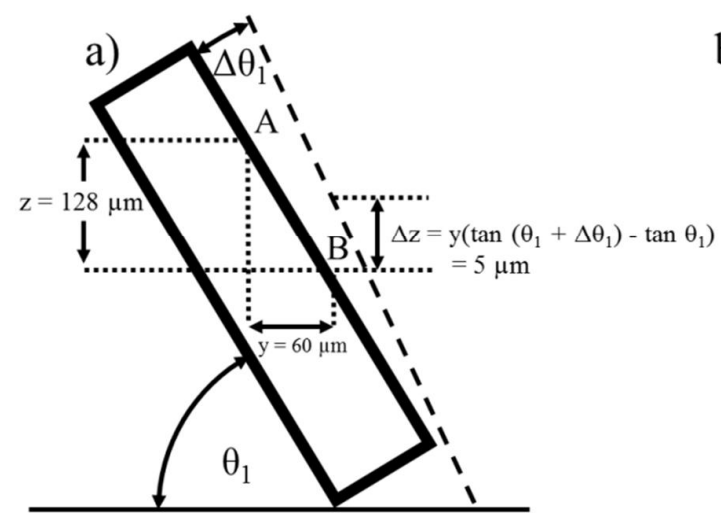

b)

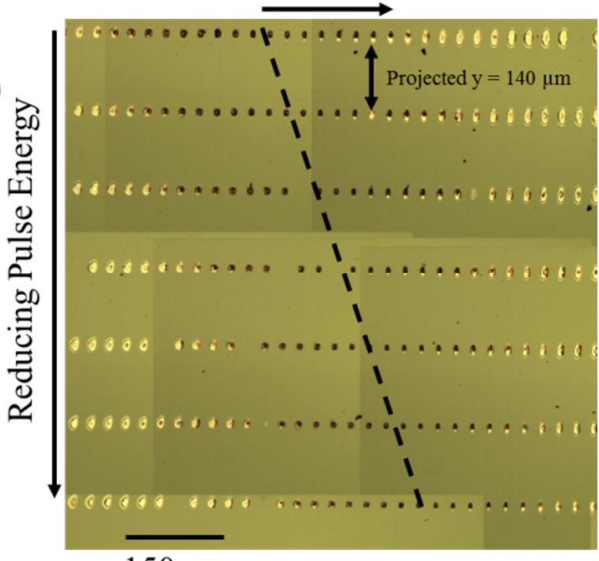

$150 \mu \mathrm{m}$

Figure 3: a) Schematic side view of a sample surface tilted at incident angle of $\theta_{1}$, with focal offset scaling as $z=y$ tan $\theta_{1}$ (point B) and focal offset error given by $\Delta z=y\left(\tan \left(\theta_{1}+\Delta \theta_{1}\right)-\tan \theta_{1}\right)$, owing to angular tilt error $\Delta \theta$. b) Optical micrograph of $\mathrm{SiO}_{\mathrm{x}}$ film exposed to array of s-polarized single laser pulses on a $30 \mu \mathrm{m}$ by $60 / \cos \left(\theta_{1}\right) \mu \mathrm{m}$ spaced grid, with varying pulse energy shown vertically between rows and focus displacement shown horizontally in the plane of the image. The best focus position, with highest fluence exposure, is indicated by the dashed line.

The grid processing pattern for a sample tilted at $\theta_{l}=65^{\circ}$ yielded the optical microscopy image of quantized ejection features as shown in Figure 3(b) for s polarized light. Maximum incident angle was limited to a sample tilt of $65^{\circ}$ with the use of a low NA lens due to geometric restrictions. Observation of colour and morphology changes in the images offered an initial indication for quantized ejection in this figure, and were used to select exposure sites for further analysis. Pulse energy was held constant on each row, decreasing in steps of $100 \mathrm{~nJ}$ from $1.6 \mu \mathrm{J}$ at top row to $0.7 \mu \mathrm{J}$ at bottom row of Figure 3, separated by $60 / \cos \left(\theta_{1}\right)=140 \mu \mathrm{m}$ spacing. Adjacent spots within each row have focal position offsets of $5 \mu \mathrm{m}$, with the tightest focusing exposure indicated by the dashed line for spots with the smallest and darkest laser modifications. 
The measured spot-to-spot separation indicated a corrected sample tilt angle of $63 \pm 1^{\circ}$ for p- polarisation and $64 \pm 1^{\circ}$ for spolarisation. The remaining alignment error arose principally from the target azimuthal uncertainty with respect to the axis of the horizontal motion stage used to shift the sample along each column in the figure. A site-to-site vertical displacement of $\mathrm{z}=128 \mu \mathrm{m}$ (Figure 3a) was required to maintain constant focal displacement and a deviation of $\sim 5 \mu \mathrm{m}$ in the intended axial focus shift was observed - labelled as $\Delta z$ in Figure 3a. Since focal displacement is varied by $5 \mu \mathrm{m}$ between features, which are spaced by $30 \mu \mathrm{m}$, we see a physical shift of $30 \mu \mathrm{m}$ when comparing the best focus of feature rows down the sample slope. The angular tilt of the best focus line in Figure $3 \mathrm{~b}$ displays this combed error.

The fluence value for each exposure spot was determined from $F=E / A$, where $\mathrm{E}$ is the pulse energy and projected beam area, A, was assessed with the above alignment corrections for spot size, $w(z)$, and tilt angle, $\theta_{l}$, according to:

$$
A=\pi w(z)^{2} / \cos \left(\theta_{1}\right)
$$

Corrections for varying on-target spot size along each row in Figure 3(b) were adjusted by Gaussian beam optic calculation.

\section{RESULTS AND DISCUSSION}

Laser exposure spots as shown in Figure 3(b) were further evaluated by SEM, with representative samples shown in Figure 4 comparing exposures with s-polarization (a-c) and p-polarization (d). For s-polarization, single layer ejection of the first segment, $\mathrm{N}_{1}$, to a depth of $240 \pm 20 \mathrm{~nm}$ can be clearly identified at a low fluence of $0.63 \mathrm{~J} / \mathrm{cm}^{2}$. Ejection of the second segment, $\mathrm{N}_{2}$, and three segment, $\mathrm{N}_{3}$ follow in (b) and (c), respectively, with small fluence increases to $0.93 \mathrm{~J} / \mathrm{cm}^{2}$ and $1.29 \mathrm{~J} / \mathrm{cm}^{2}$, respectively. Here, observed segment thicknesses of $200 \pm 20 \mathrm{~nm}$ are somewhat smaller than the expected half-wavelength expectation of $\lambda / 2 \cos \left(\theta_{i}\right) n_{\text {film }}=230 \mathrm{~nm}$, possibly owing to ablation thinning effects by the plasma disks. The features formed here at a targeted $65 \pm 2^{\circ}$ sample tilt with s- polarized light are similar to those previously reported [14] for normal incidence, (Figure 1), showing definitive evidence of quantized ejection. The beam asymmetry of 1:2.4 expected from the sample tilt is evident in both the optical microscope and SEM morphology. By controlling $\theta_{1}$, both feature asymmetry and segment thickness manipulation may be explored.

Changing laser polarization state from $\mathrm{s}$ to $\mathrm{p}$ revealed full ejection of the $1 \mu \mathrm{m}$ thick $\mathrm{SiO}_{\mathrm{x}}$ film, noted by the $1 \pm 0.1 \mu \mathrm{m}$ wall depth of the ejection site (corrected for $30^{\circ}$ observation angle) with low fluence exposure of $0.60 \mathrm{~J} / \mathrm{cm}^{2}$ as shown in Figure 4(b). Evidence of substantial substrate melting indicates that interface absorption is dominating when interference contrast is weak in the film, as anticipated by the weak 0.15 visibility of fringes in Figure 2(b) for p-

polarization. Testing of other fluence and beam size exposures in the present $1 \mu \mathrm{m}$ film did not reveal any evidence of quantized ejection on half-wavelength segmention for p- polarized light. A lower threshold for interface ejection of film is therefore noted for p-polarization. Further exploration of other angles between $0^{\circ}$ and $65^{\circ}$ may reveal a minimum fringe visibility condition for enabling interferometric quantized ejection in $\mathrm{SiO}_{\mathrm{x}}$ film.

The observations in Figure 4 categorically demonstrate the important role of laser polarization in the control of intrafilm quantized ejection. There is potential for further control in the transition between intrafilm quantized ejections with $\mathrm{s}$ polarized light and full film ejection with p- polarised light. Further study with a range of angles between 0 and $65^{\circ}$ would allow for smooth control of fringe spacing, offering precise step height adjustment in the ejected film morphology, with additional tailoring of feature ellipticity. The formation of quantized ejections at high incident angles demonstrates further that for the spot sizes $(4.6$ to $12.3 \mu \mathrm{m})$ tested here, there is sufficient multi-beam overlap within the beam area before walk-off effects would otherwise reduce the fringe visibility. Future study in testing smaller spot sizes or thicker films would help elucidate the limits of such walk-off effects. The research results may find broadly based 
industry significance where thin film processing at varying incident angles may be achieved with greater flexibility and control using today's multi-axis beam delivery tools.

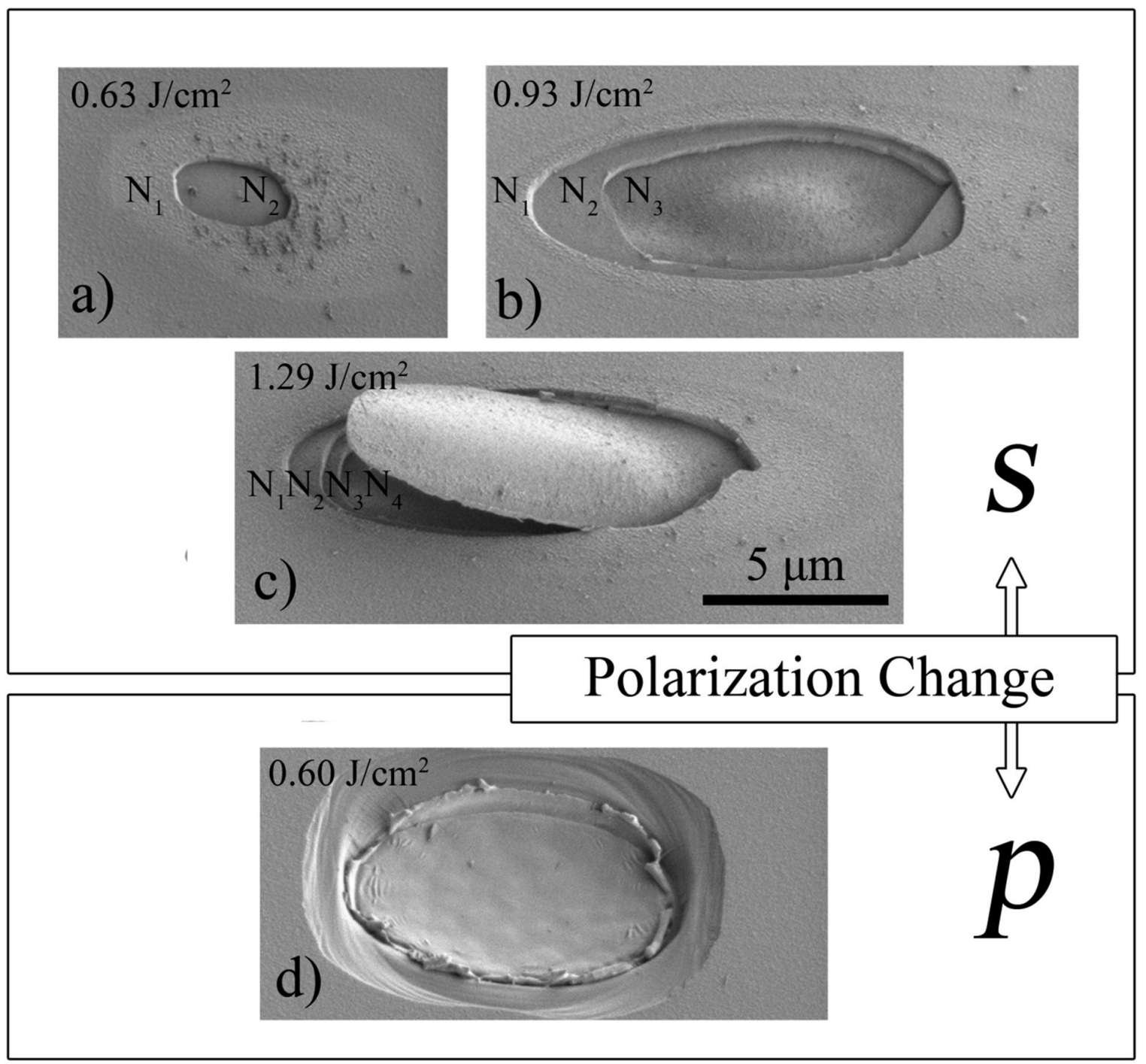

Figure 4: SEM images of $1 \mu \mathrm{m} \mathrm{SiO}$ films on silicon exposed to fluences of a) $0.63 \mathrm{~J} / \mathrm{cm}^{2} \mathrm{~b}$ ) $0.93 \mathrm{~J} / \mathrm{cm}^{2}$ and $\left.\mathrm{c}\right) 1.29 \mathrm{~J} / \mathrm{cm}^{2}$ for $\mathrm{s}$ polarized light, and d) $0.60 \mathrm{~J} / \mathrm{cm}^{2}$ for p polarized light, irradiated with single laser pulses of $515 \mathrm{~nm}$ wavelength at $\theta_{1}=\sim 65^{\circ}$ tilt angle. The $1^{\text {st }}\left(\mathrm{N}_{1}\right), 2^{\text {nd }}\left(\mathrm{N}_{2}\right)$ and $3^{\text {rd }}\left(\mathrm{N}_{3}\right)$ intra-film segments are shown to be ejected with increasing fluence exposure from a) to c). The $n_{2}$ and $n_{3}$ segment thickness of around $200 \mathrm{~nm}$, corrected for observation angle, approximately matches with the expected fringe spacing of $230 \mathrm{~nm}$. In contrast, d) shows complete film ejection at much lower fluence exposure, without evidence of quantized ejection in the film, owing to poor contrast of p-polarized interference fringes. 


\section{CONCLUSIONS}

We have developed reproducible methodology for testing the response of quantized intra-film ejection processes due to angle and polarization variation. Quantized ejections at high incident angles of $65^{\circ}$ were observed for s- polarized light, where enhanced Fresnel reflections increase the interferometric fringe contrast without deleterious beam walk-off effects in the film. In contrast, the weaker interface reflections by p- polarized light were detrimental to intrafilm processing, and interface ejection of the whole film layer was seen to dominate. The results demonstrate a facile control in the transition between intrafilm interferometric processing and interface-dominated interactions to offer multi-purpose fabrication within a single film-substrate system. A further study of intermediate exposure angles promises to open full control over ejected segment thickness and feature ellipticity. The present method more generally offers the possibility of extension of quantized ejection into new material systems through polarization and angle control beyond the Fresnel reflection limits of normally incident exposure. Development in new thin-film systems following these methods provides opportunity in advanced tailoring of surface topography to a wide range of coating applications. 


\section{ACKNOWLEDGEMENTS}

We would like to thank the Natural Sciences and Engineering Research Council of Canada and the University of Toronto for supporting this project.

\section{REFERENCES}

[1] K M Davis, K Miura, N Sugimoto, and K Hirao, "Writing waveguides in glass with a femtosecond laser," Optics Letters, vol. 21, no. 21, pp. 1729-1731, 1996.

[2] L. Shah, A Y. Arai, S M. Eaton, and P R. Herman, "Waveguide wriiting in fused silica with a femtosecond fiber laser at 522nm and $1 \mathrm{mhz}$ repetition rate," Optics express, vol. 13, no. 6, pp. 1999-2006, 2006.

[3] A Dragomir, D N Nikogosyan, K A Zagorulko, P G Kryukov, and E M Dianov, "Inscription of fiber bragg gratings by ultraviolet femtosecond radiation," Optics Letters, vol. 28, no. 22, pp. 2171-2173, 2003.

[4] A Martinez, M Dubov, A Khrushchev, and I Bennion, "Direct writing of fibre bragg gratings by a femtosecond laser," Electronics Letters, vol. 40, no. 19, 2004.

[5] A M Streltsov and N F Borrelli, "Fabrication and analysis of a directional coupler written in glass by nanojoule femtosecond laser pulses," Optics Letters, vol. 26, no. 1, pp. 42-43, 2001.

[6] R R. Gattass and E Mazur, "Femtosecond laser micromachining in transparent materials," Nature Photonics, vol. 2, pp. 219-225, 2008.

[7] M Y. Xu, J. Li, L D. Lilge, and P R. Herman, "F2- Laser patterning of indium tin oxide (ITO) thin film on glass substrate," Applied Physics A, vol. 85, no. 1, pp. 7-10, 2006.

[8] D B Chrisey, A Pique, R A McGill, J S Horwitz, and B R Ringeisen, "Laser Deposition of POlymer and Biomaterial Films," Chemical Reviews, vol. 103, no. 2, pp. 553-576, 2003.

[9] J P McDonald, A A McClelland, Y N Picard, and S M Yalisove, "Role of a native oxide on femtosecond laser interaction with silicon (100) near the damage threshold," Applied Physics Letters, vol. 86, no. 26, 2005.

[10] J P McDonald, V R Mistry, K E Ray, and S M Yalisove, "Femtosecond pulsed laser direct write production of nano- and microfluidic channels," Applied Physics Letters, vol. 88, no. 18, 2006.

[11] K. Kumar, K KC. Lee, J. Nogami J. Li, N P. Kherani, and P R. Herman, "Quantized structing of transparent films with femtosecond laser interfererence," Nature: Light Science \& Applications, vol. 3, no. 3, 2014.

[12] K Kumar, K K Lee, J Nogami, and P R Herman, "Interferometric Laser Processing," US20160158886 A1, July 22, 2014.

[13] S. Ho, K. Kumar, K K C. Lee, J. Li, and P R. Herman, "Interferometric femtosecond laser processing for nanostructuring inside thin film," Advanced Optical Techniques, vol. 3, no. 5-6, pp. 499-513, 2014.

[14] S. Ho, M. Domke, D M. Roper, H. Huber, and P R. Herman, "Femtosecond Laser Quantized Structing in thin SiOx Film," CLEO: Conference on Lasers and Electro-Optics, 2016.

[15] M. Haque, K K C. Lee, S Ho, L A. Fernandes, and P R. Herman, "Chemical-assisted femtosecond laser writing of lab-in-fibers," Lab on a Chip, vol. 14, no. 19, pp. 3817-3829, 2014.

[16] M. Haque, "Chemical-assisted Femtosecond Laser Writing of Lab-in-fiber Sensors," Doctorial Dissertation, University of Toronto, 2014. 